\title{
Trabajar en grupo y aprender haciendo: desarrollo de competencias clave para los estudiantes del siglo XXI.
}

\section{María Jano Salagre ${ }^{\mathrm{a}}$ S Salvador Ortiz Serrano}

${ }^{a}$ Profesora del Departamento de Economía Aplicada de la Universidad Autónoma de Madrid, dolores.jano@uam.es y ${ }^{b}$ Profesor del Departamento de Economía Aplicada de la Universidad Autónoma de Madrid, salvador.ortiz@uam.es

\begin{abstract}
In recent times, a new competence that combines two verbs: learn and teach, is gaining relevance and is undoubtedly essential in a world of information overflow, uncertainty and drastic changes. Our students have to prepare to adapt quickly to changes and need to develop an active attitude. They have to learn to work as a team, collaborating to detect problems and teach others how to solve them, share knowledge and communicate in an effective way. These are the skills that we intend to expound on in the presented experience.

The objective of the communication is to describe the experience and assess the effect that the group may have on the individual's results. The results show that, discounting the effect that previous skills and knowledge may have, group work contributes to improving the individual grades of the student, whether she/he is assessed as a whole, or assessed individually.
\end{abstract}

\section{Keywords:}

Active learning, Cooperative Learning, Higher Education, Statistics, Learning Outcom

\section{Resumen}

En los últimos tiempos gana relevancia una nueva competencia que conjuga dos verbos: aprender y enseñar, y que sin duda es imprescindible en un mundo de sobreabundancia de información, incertidumbre y cambios drásticos. Nuestros alumnos han de prepararse para adaptarse rápidamente a los cambios, y necesitan desarrollar una actitud activa. Tienen que aprender a trabajar en equipo, colaborando para detectar problemas y enseñar a otros cómo resolverlos, compartiendo conocimientos y comunicando de una manera eficaz. Estas son las habilidades que se pretenden desarrollar en la experiencia que se presenta.

El objetivo de la comunicación es describir la experiencia y valorar el efecto que puede tener el grupo en los resultados del individuo. Los resultados muestran que, descontando el efecto que pudieran tener las habilidades $y$ conocimientos previos, el trabajo en grupo contribuye a mejorar las calificaciones individuales del estudiante, tanto si es valorado en su conjunto, como si se evalua individualmente. 
Palabras clave: Aprendizaje Activo, Aprendizaje cooperativo, Educación Superior, Estadística, Resultados de Aprendizaje

\section{Introducción}

Según afirmaciones basadas en la "pirámide del aprendizaje” enseñar es la mejor manera de aprender, habitualmente se dice que los aprendices retienen el 90\% de lo que aprenden cuando se lo enseñan a otros o lo utilizan de inmediato. No obstante son porcentajes discutidos por algunos autores (Lalley; J.P. y Miller; R.H. 2007) y habría que tener en cuenta el contexto en que se realiza la experiencia, la manera de llevarla a cabo, la implicación de los propios estudiantes y cómo se mide el aprendizaje.

El reto como profesores en esta nueva era es conseguir que el alumno asuma un papel activo, se haga cargo de su propio aprendizaje, lo comparta con otros y esté dispuesto a seguir como aprendiz a lo largo de su vida, desarrollando su "learnability" o habilidad de aprender rápida y eficazmente. Duran (2014) habla de "aprenseñar” y aprender en 3D; a lo largo, ancho y en lo profundo de nuestras vidas, que va más allá del aprendizaje permanente ó Lifelong Learning. Se trata no sólo de promover un enfoque activo, sino que además, hay que ayudarles a desarrollar otras competencias clave en su empleabilidad, en un mundo en el que la información que tendrán que manejar es amplia, heterogénea, compleja, altamente especializada y donde el trabajo conjunto con profesionales de diferentes áreas será cada vez más común. Por tanto hay que darles oportunidades para trabajar en grupo, resolver problemas y transmitir la información a otros de manera eficaz.

Con el aprendizaje activo se trataría de acabar con la enquistada y tradicional pasividad del alumno, generando contextos que promuevan situaciones para lograr un aprendizaje profundo, en los que los protagonistas sean los estudiantes que se relacionan entre ellos y con el profesor. Con el trabajo cooperativo, se trataría de fomentar aprendizajes a través del trabajo guiado y tutelado de grupos heterogéneos de estudiantes, a los que se proponen tareas que supongan un reto abordable para ellos, y en el que pongan en práctica los conocimientos teóricos adquiridos en situaciones concretas. Con ello los alumnos van logrando mayores grados de autonomía, responsabilidad y compromiso. En todo este proceso, el apoyo y la guía del profesor es imprescindible, de manera que una adecuada retroalimentación del proceso les permitirá aprender también de los errores cometidos. Hulsizer and Woolf (2008) comparten las mejores prácticas en la docencia en estadística que promueven situaciones de aprendizaje activo, cooperativo y basado en problemas. Además, existen innumerables experiencias en otros campos como Marketing Chad (2012), Chapman, Meuter, Toy, y Wright (2010); Administración de Empresas Kidder y Bowes-Sperry (2012) o en Economía Hettler (2015).

En cursos pasados se pusieron en marcha iniciativas para fomentar el aprendizaje activo, tareas para que los alumnos aprendan haciendo, resolviendo ejercicios propuestos por el profesor, preparando y exponiendo un tema completo y participando en una amplia batería de actividades que involucran al estudiante y le ayudan a aprender de manera continua y no sólo unas semanas antes del examen final (Jano y Ortiz 2017a y 2017b). Sin embargo hasta

(cc) EY-NC-ND 2018, Universitat Politècnica de València

Congreso IN-RED (2018) 
ahora no se habían explorado las posibilidades que ofrece el trabajo en grupo. Además de las propuestas en las que los alumnos asumen el rol del profesor, inventando problemas y explicando en clase cómo resolverlos al resto de compañeros, se han ido incorporado tímidamente actividades para fomentar el aprendizaje cooperativo y colaborativo en la docencia en la asignatura de Estadística Teórica.

La estructura de la comunicación se organiza en cuatro partes. Una primera parte donde se detallarán los objetivos de la presente comunicación, a continuación se explicará cómo se ha desarrollado la innovación, para continuar con un análisis de los resultados obtenidos y por último, se hará una reflexión sobre las lecciones aprendidas.

\section{Objetivos}

Es conocido que los estudiantes utilizan dinámicas para trabajar en grupo que no resultan adecuadas para el aprendizaje, como repartartirse las tareas, ir de polizón en el grupo, o no no asumir el compromiso individual necesario para lograr que el grupo logre los mejores resultados. Uno de los objetivos de nuestra propuesta es experimentar procedimientos que eviten esas situaciones y que fomenten que aquellos alumnos con más habilidades puedan facilitar el camino a los que se encuentran con más dificultades.

Un segundo objetivo, relacionado con el anterior, pretende comprobar si, como sería deseable, las calificaciones individuales se ven afectadas positivamene por la valoración que se hace del trabajo del grupo en su conjunto, y por el trabajo que el alumno hace con el apoyo del grupo, pero del que responde individualmente.

Además, hay otro componente importante en la valoración de los resultados individuales del estudiante que habría que considerar. No todos los alumnos parten del mismo punto de salida en la carrera, algunos tienen un bagaje y unas habilidades que les favorecen, una mejor predisposición en asignaturas cuantitativas o tienen hábitos de trabajo y productividad distintos. Por tanto, se contrastará si los resultados previos en una asignatura que se imparte en el curso anterior por el Departamento, la Estadística Descriptiva, es un factor significativo a la hora de predecir los resultados individuales del estudiante.

\section{Desarrollo de la innovación}

\subsection{Contexto en que se lleva a cabo}

Se trata de la docencia de la asignatura Estadística Teórica en dos grupos en el grado en ADE (Administración y Dirección de Empresas), de 6 créditos, en la que se explican cuestiones de probabilidad e inferencia, y que se imparte por los mismos profesores en el primer cuatrimestre del curso. En total se refiere a 110 alumnos, de los que sólo 11 están en segunda o posteriores convocatorias. En el análisis de los resultados se descartarán a estos últimos estudiantes, puesto que la mayoría optó porque su evaluación continua fuera a través de dos controles a lo largo del curso, y por otro lado ya han cursado la asignatura y parten de una situación diferente al resto. 
La nota final se obtiene en un $60 \%$ mediante un examen final, y un $40 \%$ por la evaluación continua a lo largo del curso. Ese $40 \%$ se reparte de la siguiente manera: $20 \%$ en dos controles escritos, $10 \%$ un trabajo en grupo en Excel (5\% la presentación de un informe conjunto del grupo y $5 \%$ una prueba en las aulas de informática) y un $10 \%$ de valoración del trabajo autónomo mediante la realización de una colección de ejercicios y la propuesta, resolución y exposición en clase de un problema original que se formula en grupo (5\% exámenes breves sobre los ejercicios propuestos y $5 \%$ la defensa individual de un ejericicio original). Además a lo largo del curso se propusieron abundantes tareas y actividades a realizar, algunas en clase y otras a través de la plataforma, que bonificaban la nota de evaluación continua en un máximo de 1,5 puntos.

\subsection{Propuesta de trabajo en grupo}

Se formaron diez grupos de cinco alumnos, heterogéneos en base a sus calificaciones en estadística despcriptiva. Se ordenaron las notas y se iban asignando a los grupos, garantizando que en todos ellos hubiera estudiantes con notas altas, intermedias y bajas.

Cada grupo debía trabajar sobre una base de datos distinta a partir de los microdatos que publica el INE (Instituto Nacional de Estadística). Se trataba de trabajar en la elaboración de un informe sobre un caso real que aborda de manera aplicada todos los aspectos teóricos explicados en la asignatura. En concreto, se analizaban las diferencias en los gastos por día de los turistas de distintas comunidades autónomas, según motivo de viaje. El informe debía dar respuesta a una serie de preguntas relativas a obtención de las muestras, estimación puntual y por intervalos, así como contrastes de diferencia de medias, unilaterales y bilaterales. Como apoyo para la realización de la práctica se dieron varias clases en el aula de informática, se prepararon varios vídeos que se pusieron a disposición de los estudiantes en el canal de youtube que aparece citado en la bibliografía, y se llevaron a cabo diversas tutorías para el seguimiento de los trabajos.

Pero además, el grupo debe inventar un ejercicio práctico referido a una parte del temario de la asignatura asignado al azar. El grupo trabaja junto para logar un enunciado original y proponer su resolución. La intención es crear un documento con los problemas propuestos y resueltos y ponerlo a disposición del resto de alumnos en un repositorio en Moodle.

Aunque el peso sobre la nota final de la valoración del grupo en su conjunto es muy bajo, sólo un 5\%, todas estas actividades están diseñadas para mejorar el aprendizaje y por tanto para conseguir buenos resultados en el examen final, que si tiene un peso importante en la nota final (60\%). Y no hay que olvidar que hay un porcentaje de la evaluación (un 5\%) que obtiene el alumno individualmente, pero al amparo del grupo. Sabían que podían repartirse el trabajo como consideraran conveniente, pero que todos debían saber defenderlo integramente, porque tendrían que demostrarlo individualmente en diversas pruebas.

\subsection{Propuestas de trabajo en grupo con valoración individual}

La valoración individual del trabajo en grupo tiene tres componentes:

(cc) BY-NC-ND 2018, Universitat Politècnica de València

Congreso IN-RED (2018) 
- La resolución de unos 16 problemas que iban haciendo por su cuenta a medida que se avanzaba en los contenidos teóricos del curso, y que eran valorados mediante una breve prueba en clase sobre algunos apartados de los problemas propuestos. Podían colaborar entre ellos antes de la prueba para solucionar los ejercicios, pero finalmene eran evaluados por escrito de manera individual. Más tarde se publicaban las soluciones para que ellos mismos comprobaran y detectaran los errores cometidos.

- La elaboración por grupos de un problema original, que tendrían que resolver y enviar al profesor para que diera su visto bueno, y que posteriormente explicaban a la clase. El profesor elige al azar qué parte tendrá que explicar cada uno.

- En la tercera, cada alumno tendría que resolver en las aulas de informática algún apartado del trabajo en grupo presentado y comentar brevemente los resultados obtenidos.

\section{Resultados obtenidos}

\subsection{Definición de las variables}

Aunque las calificaciones no siempre son un fiel reflejo de lo que sabe el estudiante, y de lo significativos que son esos aprendizajes, se utilizarán como indicadores para poder valorar cuantitativamente los resultados de la experiencia. Las variables utilizadas se resumen en la Tabla 1. Se valoran tres aspectos, el resultado individual, medido a través de la calificación en el examen final que incluye preguntas sobre todo lo trabajado en el curso. El resultado del trabajo en grupo, recogida a través de la calificación conjunta obtenida en el informe. El resultado del aprovechamiento del individuo dentro del grupo, recogido a través exposición que hace el alumno en clase de algún apartado del ejercicio original propuesto por el grupo. También se tendrá en cuenta, como referencia previa la calificación en Estadística Descriptiva.

Tabla 1. Variables analizadas.

\begin{tabular}{|l|}
\hline \multicolumn{1}{|c|}{ Etiquetas y codificación de variables } \\
\hline Nota del Examen Final: INDIVIDUAL \\
\hline Nota del Individuo en la Exposición del problema original: EN EL GRUPO \\
\hline Nota del Informe Elaborado por el Grupo: GRUPO \\
\hline Calidad de la participación del grupo: \\
- Mala GRM: Nota de Grupo menor a cinco (Grupo de referencia) \\
- Regular GRR: Nota de Grupo mayor o igual a cinco y menor de nueve \\
- Buena GRB: Nota de Grupo mayor o igual a nueve \\
\hline Calidad de la exposición individual del problema original: \\
- Mala ENGRM Nota de Grupo por debajo de la Mediana (Grupo de referencia) \\
- Buena ENGRB Nota de Grupo por encima de la Mediana \\
\hline Nota en la asignatura del curso pasado Estadística Descriptiva: DESCRIPTIVA \\
- Nivel Previo Bajo: Nota en Descriptiva por debajo de la Mediana \\
- Nivel Previo Alto: Nota en Descriptiva por encima de la Mediana \\
\hline
\end{tabular}

Fuente: Elaboración propia 
Además, en algunas ocasiones, y para facilitar la interpretación, se recodifican las variables referidas a las notas en tramos o escalas de calificacion. Se definirán tres categorías según la calidad en el trabajo en grupo; mala (menos de cinco puntos), regular (entre cinco y nueve puntos) y buena (nueve o más puntos). En el caso de las calificaciones en función de la calidad del trabajo individual realizado al amparo del grupo y de la nota previa de Estadística, sólo se definirán dos grupos (según estén por debajo o por encima de la mediana).

\subsection{Análisis de los resultados obtenidos}

Respecto a la valoración de la propuesta de innovación, se pueden destacar dos cuestiones. En primer lugar, el poco tiempo que disponen profesores y alumnos para una asignatura compleja y que se construye sobre los conocimientos que van adquiriendo y que requiere un proceso de consolidación de lo que se va construyendo. Se trata de 6 créditos en el primer cuatrimestre, lo que supone 3 horas de clase semanales para abordar probabilidad e inferencia estadística. Las últimas semanas del curso, cuando han de finalizar el trabajo y conseguir una visión global de la utilidad de la asignatura, los alumnos tienen mucha presión por entregar tareas y hacer exámenes en todas las asignaturas, se ven sobrepasados y muchos abandonan la asignatura para la convocatoria extraordinaria.

En segundo lugar, las calificaciones individuales son muy importantes para ellos, porque está en juego poder acceder al Programa de Cooperación Educativa, que consideran atractivo de cara a su futuro laboral. No quieren perder tiempo, ni que las notas en grupo puedan perjudicar sus expectativas.

La presión del tiempo es quizá uno de los factores más difíciles de superar en el proceso. Los alumnos aprenden, pero se quejan en las encuestas de valoración de este curso, de la excesiva carga de trabajo y sobre todo del elevado número de pruebas a lo largo del curso.

Quizá una alternativa podría ser utilizar la tecnología para no perder tiempo en clases y poder valorar de manera adecuada y con garantías la participación del alumno en el grupo. Por ejemplo, mediante la exposición individual del trabajo y de los problemas en vídeos, que se compartirán con todos los estudiantes y podrán ser vistos y valorados por todos ellos. Aunque también hay resistencias de algunos alumnos a exponerse públicamente de esa manera, ya que les causa mucha tensión, y a ser evaluados por sus compañeros, ya que puede generar conflictos entre ellos.

No obstante, valoran muy positivamente la cantidad de recursos y materiales de que disponen, y el trabajo en Excel, de cara a su futuro profesional.

Otra dificultad en el proceso es la gestión de los conflictos y dificultades que surgen en el trabajo con grupos. Un aspecto clave es que los profesores aprendan técnicas y procedimientos para mejorar las dinámicas y evaluación del trabajo en grupo, y que los alumnos también aprendan a gestionar conflictos, habilidad que les resultará clave e imprescindible su futuro profesional. Todo esto una vez más requiere tiempo y disposición de unos y otros, que a veces es difícil de encontrar.

(cc) EY-NC-ND 2018, Universitat Politècnica de València

Congreso IN-RED (2018) 
Respecto a la relación entre los resultados individuales en función del grupo y del desempeño del estudiantes al amparo del grupo y de sus conocimientos previos, en la Figura 1 se puede comprobar que el bagaje y habilidades previas que el estudiante trae, tiene un efecto en las calificaciones en los tres indicadores propuestos, que se refieren a: su trabajo individual (nota del examen final), su trabajo dentro del grupo (nota de la exposición del problema original) y el trabajo del grupo (nota del informe de grupo presentado). En todos ellos se observan mejores notas en este curso para los alumnos que están por encima de la mediana en Estadística Descriptiva. En media algo más de dos puntos en el Examen Final, un punto en la nota del Grupo y más de medio punto de la exposición individual del Trabajo en el Grupo. Por tanto, tendrá sentido descontar este efecto al estudiar los resultados de los alumnos en este curso.

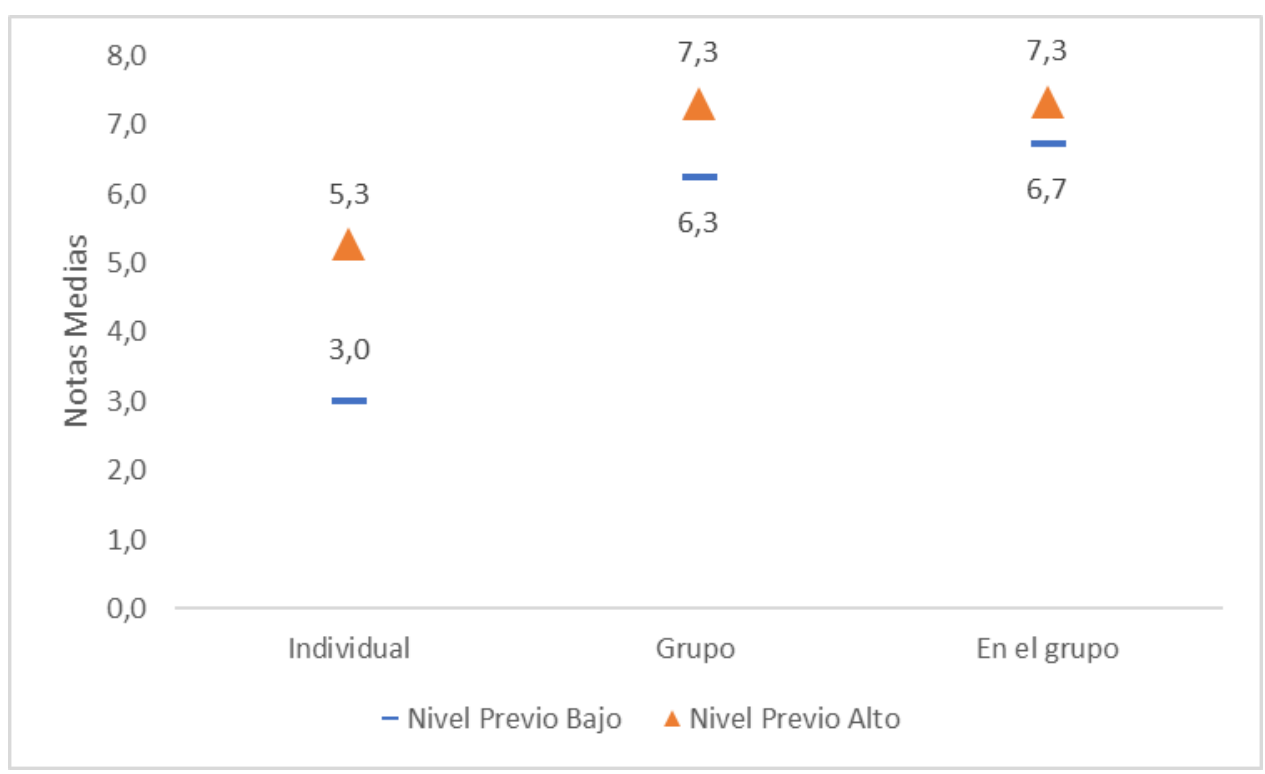

Fig. 1. Diferencias en notas medias del estudiante en este curso según sus conocimientos previos

Pero aunque los grupos son heterogéneos en función de las notas, y en todos ellos hay estudiantes de todos los niveles, hay variabilidad en las calificaciones de los informes. Si se analizan diferencias en los resultados según la calidad del trabajo del grupo dividido en tres categorías, Mala, Regular o Buena, se observa que hay un efecto en las notas. Esto es lo que se presenta en la Figura 2, donde se aprecia, en general, que hay una relación positiva entre la nota del trabajo en grupo y las notas individuales obtenidas por el alumno. Además, en todos los grupos se observan unas notas más altas cuando se cuenta con el apoyo del grupo repecto a las notas individuales en el examen final, si bien las diferencias se van haciendo más pequeñas. Es decir, a nivel descriptivo se observa que, en general, cuanto mejor es el grupo, mejor son los resultados individuales de sus miembros. 


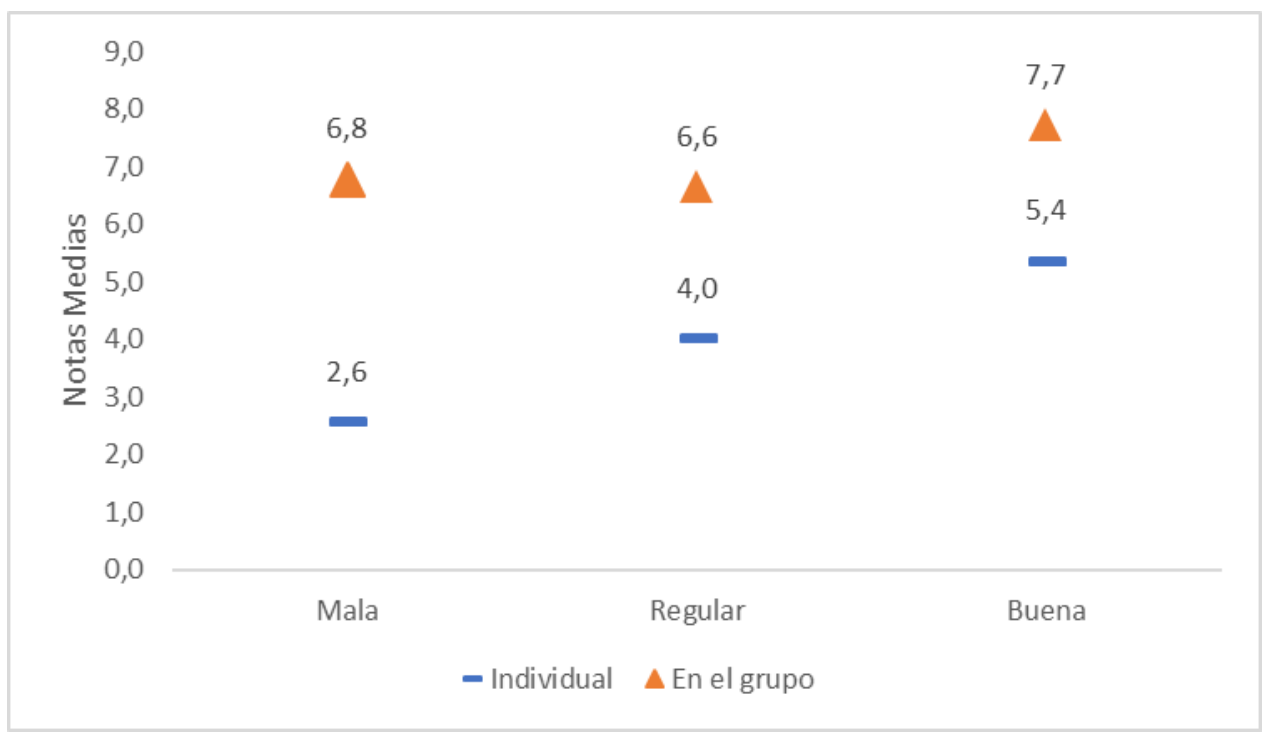

Fig. 2 Calificaciones del alumno según calidad del trabajo en grupo.

Esta relación positiva y significativa se confirma al calcular los coeficiente de correlación entre la nota individual del examen con las notas del grupo y del individuo dentro del grupo, que en ambos casos vale 0,34.

Pero para valorar el efecto en las diferencias en la nota final, se propone un modelo de regresión que explique el resultado individual a partir de los resultados del trabajo en grupo, del trabajo individual con apoyo del grupo y de sus resultados previos. Como se aprecia en los resultados del primer modelo (1) hay una relación positiva y significativa de cada uno de estos indicadores sobre el resultado individual. De manera que, descontando el efecto de las demás variables del modelo, un aumento de las notas en las que participa el grupo se traduce en aumentos en la nota individual.

$$
\begin{gathered}
\text { INDIVIDUAL }=-4,43+0,22^{* *} \text { GRUPO }+0,20^{*} \text { EN EL GRUPO }+0,97^{* *} \text { DESCRIPTIVA (1) } \\
\mathrm{r}^{2}=0.356 \\
\\
\text { * coeficiente significativo para un } 5 \% \text { de nivel de significación } \\
\end{gathered}
$$

Para percibir mejor la magnitud de los efectos se utilizará un modelo de análisis de la covarianza, en el que se propone como variable a explicar el rendimiento individual, y como variables explicativas la nota previa en descriptiva como covariable y dos factores. Por un lado, el factor grupo definido en tres categorías, y por otro lado, el factor individual con apoyo del grupo, recodificada en dos categorías, como se indicaba en la Tabla 1 de descripción de variables. Se consideran como categorías de referencia la de las peores calificaciones. Como se aprecia en los resultados del segundo modelo (2) todos los coeficientes son significativos y refrenda la influencia del grupo en los resultados individuales.

$$
\begin{aligned}
& \text { INDIVIDUAL }=-3,1+1,6^{* *} \text { GRR }+2,03^{* *} \text { GRB }+1,18^{*} \text { ENGRB }+0,90^{* *} \text { DESCRIPTIVA (2) } \\
& \text { (Modelo 2) } r^{2}=0.41 \\
& \text { * coeficiente significativo para un 5\% de nivel de significación } \\
& \text { ** coeficiente significativo para un 1\% de nivel de significación }
\end{aligned}
$$

(cc) EY-NC-ND 2018, Universitat Politècnica de València 
De la interpretación de los coeficientes estimados por el modelo se observa que, manteniendo todo lo demás constante, si el grupo es regular, la nota media en el examen individual es 1,6 puntos mayor que si el grupo es malo, y si el grupo es bueno, el efecto es aún más claro, obteniendo 2,03 puntos más en el examen final que los que de peor desempeño. De manera análoga, los que tienen buena nota individual del trabajo realizado en grupo, consiguen en media un punto más en el examen final que los que hacen una exposición pobre del problema original. Se podría decir que obtener buenos resultados en grupo, en general, ayudará a que cada uno de sus miembros obtenga también mejores resultados a nivel individual.

\section{Conclusiones}

El mundo laboral al que se enfrentarán nuestros estudiantes es cada vez más complejo y requiere el desarrollo de habilidades que les permita mejorar el acceso puestos de trabajo de calidad. Además de aprender a lo largo de su vida, se verán obligados a colaborar con otros para resolver problemas complejos y multidisciplinares, compartiendo sus conocimientos. Para preparar a nuestros estudiantes ante ese reto, este curso se han propuesto diversas actividades que promueven su creatividad y espíritu colaborativo.

El análisis de los resultados confirma que el grupo contribuye a mejorar los resultados individuales, por lo que es recomendable promover y ampliar las actividades que se realizan en grupo y darles protagonismo a ellos en su aprendizaje, animándoles a proponer y resolver problemas.

Como debilidades y aspectos a mejorar, hay que destacar que no se han tenido en cuenta las valoraciones que los estudiantes hacen de esta propuesta, más allá de los comentarios que hacen en las encuestas oficiales del profesor y de la asignatura. Tampoco se ha recogido información sobre las dinámicas que surgían en el trabajo en grupo, que quizá se pudiera haber hecho a través de la elaboración de actas virtuales en las que registren las fechas de reunión, un resumen de aspectos tratados, reparto entre sus miembros de tareas, y responsabilidades, valoración entre ellos de su trabajo en el grupo o asuntos relacionados con la gestión de conflictos en el seno del grupo.

Como reflexión final, estamos convencidos de la importancia que tiene para nosotros como profesores y para nuestros estudiantes aprender a trabajar en grupo y comunicar los resultados de manera atractiva y eficaz, como competencias imprescindibles para el futuro. Consideramos que a pesar de las dificultades y resistencias a cooperar, a pesar del tiempo que hay que dedicar a organizar y gestionar bien los grupos, a pesar de los temores que surgen por la posibilidad de que afecte a los resultados del individuo; las ventajas de aprender a gestionar los conflictos y de aprovechar las sinergias que se producen, compensan con creces esos posibles inconvenientes. Merece la pena trabajar para crear un entorno en el que profesores y estudiantes experimentemos la sabiduría que encierra el proverbio "Si quieres ir rápido, camina solo; pero si quieres llegar lejos, camina acompañado" 


\section{Referencias}

DURAN, D. (2014). Aprenseñar. Evidencias e implicaciones educativas de aprender enseñando. España: Narcea.

BARRICK, M. R., BRADLEY, B. H., \& COLBERT, A. E. (2007). "The moderating role of Top Management Team interdependence: implications for real teams and working groups”. Academy of Management Journal, 50(3), 544-557.

CHAD, P. (2012). "The Use of Team-Based Learning as an Approach to Increased Engagement and Learning for Marketing Students: A Case Study”. Journal of Marketing Education, 34 (2), 128-139.

CHAPMAN, K. J., MEUTER, M. M., TOY, D. y WRIGHT, L. K. (2010). “Are student groups dysfunctional? Perspectives from both sides of the classroom”. Journal of Marketing Education, 32, 39-49.

HETTLER, P.L. (2015). “Active Learning in Economics: Increasing Student Engagement, Excitement and Success”. International Atlantic Economic Society, Vol. 21 pp. 357-360

HULSIZER, M.R. y WOOLF, L.M. (2008) A guide to Teaching Statistics: Innovations and Best Practices. Wiley-Blackwell.

JANO, M. y ORTIZ, S. (2017a). “Aprender enseñando. Una experiencia para desarrollar la quinta competencia”. Universidad Politécnica de Valencia. Tercer Congreso Nacional de Innovación Educativa y Docencia en Red IN-RED.

JANO, M. y ORTIZ, S. (2017b). “ « Buffet libre » como experiencia de aprendizaje en la asignatura de Estadística”. REDU Revista de Docencia Universitaria, Vol. 15 (2) julio-diciembre pp. 179-192. https://doi.org/10.4995/redu.2017.6760

KIDDER, D. L. y BOWES-SPERRY, L. (2012): " Examining the Influence of Team Project Design Decisions on Student Perceptions and Evaluations of Instructors " Academy of Management Learning \& Education, 11 (1), 69-81.

LALLEY, J. P \& MILLER, R.H. (2007): "The leaming pyramid: Does it point teachers in the right direction?" Education 128(l):64-79.

https://www.youtube.com/channel/UCqkM4FQL-p9G-liqitrzgyg Canal de videos de Estadística Teórica

(cc) EY-NC-ND 2018, Universitat Politècnica de València

Congreso IN-RED (2018) 